\title{
Welding Process Tracking Control Based on Multiple Model Iterative Learning Control
}

\author{
Xiaoli Li $\left(\mathbb{D},{ }^{1,2,3}\right.$ Jian Liu $\left(\mathbb{D},{ }^{1}\right.$ Linkun Wang, ${ }^{4}$ Kang Wang $\left(\mathbb{D},{ }^{1}\right.$ and Yang Li ${ }^{5}{ }^{5}$ \\ ${ }^{1}$ Faculty of Information Technology, Beijing University of Technology, Beijing 100124, China \\ ${ }^{2}$ Beijing Key Laboratory of Computational Intelligence and Intelligent System, Engineering Research Center of Digital Community, \\ Ministry of Education, Beijing 100124, China \\ ${ }^{3}$ Beijing Advanced Innovation Center for Future Internet Technology, Beijing University of Technology, Beijing 100124, China \\ ${ }^{4}$ Instrumentation Technology \& Economy Institute, Beijing 100055, China \\ ${ }^{5}$ School of international studies, Communication University of China, Beijing 100024, China \\ Correspondence should be addressed to Xiaoli Li; lixiaolibjut@bjut.edu.cn
}

Received 24 January 2019; Revised 12 April 2019; Accepted 18 April 2019; Published 16 May 2019

Academic Editor: Ines Tejado Balsera

Copyright ( 2019 Xiaoli Li et al. This is an open access article distributed under the Creative Commons Attribution License, which permits unrestricted use, distribution, and reproduction in any medium, provided the original work is properly cited.

\begin{abstract}
The welding of the same parts has same welding trajectory, so welding process has strong repeatability. In this paper, aiming at the repeatability of welding process, an iterative learning controller is designed to achieve the control of weld quality. Due to the extremely variable welding environment and the presence of noise interferences and load disturbances, it is easy to cause the jumping change in parameters and even the structure of the welding system. Therefore, the idea of multiple model adaptive control (MMAC) is introduced into iterative learning control (ILC), and a multiple model iterative learning control (MMILC) algorithm is designed according to model of weld pool dynamic process in gas tungsten arc welding (GTAW). Besides, the convergence of the algorithm is analyzed for two cases: fixed parameters and jumping parameters. It turns out that the MMILC can not only utilize the repetitive information effectively in the welding process to achieve high precision tracking control of weld seam in limited time interval, but also realize the multiple model switching according to different working conditions to improve the welding quality.
\end{abstract}

\section{Introduction}

Welding is an important process in modern industrial production. It is an operation method of joining two or more base metals into a whole body through welding materials under high temperature or high pressure conditions. As an important welding technology, gas tungsten arc welding (GTAW) is mainly used to weld easily oxidized alloys, stainless steel, and other refractory active metals, and it is also widely used in aerospace and many other industrial fields. During the welding process, an arc is generated between the tungsten electrode and the base material for welding, and at the same time, argon gas, which is used as a shielding gas to isolate air, is ejected continuously from welding torch muzzle to form a protective layer around the arc. In this way, high quality welds can be obtained. Nowadays, high quality and high efficiency are pursued in industrial production, and welding automation and robotization have become the trend of development. Improvement of welding quality is primary task of welding production automation. Therefore, the research on tracking control of welding process has practical significance.

The tracking control problem of GTAW process has always been a hot topic in the field of welding research, and many global famous scholars have done a lot of research about it. In [1], some basic concepts relating to neural networks and how they can be used to model weld-bead geometry in terms of the equipment parameters selected to produce the weld are explained. The performance of neural networks for modeling is evaluated using actual welding data. It is concluded that the accuracy of neural network modeling is fully comparable with the accuracy achieved by more traditional modeling schemes. In arc welding processes, because of the complexity and nonlinearity of heat transfer phenomena, it is often difficult to design an effective control system based upon an exact mathematical model. In [2], a control system is designed 
to comprise a combined structure of the fuzzy SOC with conventional PD control for improving the system's stability. Based on an analysis of accepted adaptive algorithms, an adaptive generalized predictive decoupling control scheme was constructed for GTAW process in [3]. In [4], a neurofuzzy model is used to model the welding process. Based on the dynamic fuzzy model, a predictive control system has been developed to control the welding process. Experiments confirmed that the developed control system is effective in achieving the desired fusion state despite the different disturbances. In [5], a PID controller was designed for the GTAW dynamic system model, and the Smith predictor was adopted to compensate time-delay at starting-welding. In order to achieve high quality control of the back weld width and the topside height of weld pool at the same time, a multi-input and multi-output adaptive fuzzy controller was designed by adding the fuzzy control to the adaptive controller to ensure the welding quality and precision [6]. In [7], a new type of fuzzy controller was designed by combining fuzzy control, neural network, and genetic algorithm in the control of GTAW process. Compared with traditional fuzzy control, the new controller had achieved better control effects. In [8], a nonlinear composite controller was designed for GTAW process. The peak current of the welding was adjusted according to the Hammerstein model, and the wire feeding speed was adjusted based on the fuzzy logic, which ensures that the back weld width and the topside height of weld pool were formed stably at the same time during the welding process. Since the precise mathematical model of welding process cannot be established, it is difficult to obtain satisfactory results by using classical and modern control theory. To overcome this problem, some researchers adopt model-free adaptive control (MFAC) to study the dynamic process of GTAW in [9]. Model-free adaptive controllers for singleinput single-output, multi-input single-input, and multiinput multi-output model of welding process were designed, respectively, and good control results were achieved. In [10], the adaptive sliding mode control algorithm was applied to the automatic welding system. By changing the length of welding arc to control the heat that transferred to the welding workpiece, the control requirements were well met. In order to imitate the estimation and decision-making process of skilled welders, an adaptive neurofuzzy inference system (ANFIS) was used to establish the model that related the back weld width to the welding current and speed, and a model predictive control was used to follow a trajectory to reach the desired back weld width [11]. Although some achievements have been made in the above research, for welding process, which has multivariable, nonlinear, time-varying parameters and contains many uncertain factors and constraints, it is almost impossible to obtain an accurate mathematical model. Therefore, it is still difficult to achieve complete tracking control of welding process.

In practical production, the process of automatic welding is a repetitive task. The repeatability is mainly reflected in the same welding workpiece and the same welding trajectory. However, the existing control methods have not considered how to use the repetitive information of welding process to improve the tracking performance of the system output.
For repetitive systems, iterative learning control (ILC) [1215] is an effective control method. The algorithm does not rely on the accurate mathematical model of the dynamic system, and it can achieve expected output trajectory tracking in limited time and interval with little prior knowledge and computational effort. The working principle of ILC is to use the previous iteration of the input information and the output error to modify control signal and gradually make the actual output converge to the desired output. In recent years, iterative learning control has not only made great progress in theory, but has also been widely used in many industrial fields [16-18], such as robotics, power, and industrial process control.

For controlled objects whose system parameters do not change or change slowly, ILC can achieve good control effects. However, in practical welding production process, due to the extremely variable welding environment and the presence of noise interference and load disturbance, it is easy to cause the jumping change in parameters and even the structure of the welding system. In this case, ILC may not perform well and lead to the number of iterations increase, and more waste products are produced. According to the ILC theory, for the unknown dynamic systems, as long as convergence condition is satisfied, uniform tracking performance can be guaranteed even with partially known or estimated model. Though the exact model is not required, number of iteration for ILC with estimated model will increase compared with ILC with exact model. Multiple model adaptive control (MMAC) [19-21] is an effective method for the system with jumping parameters. According to the MMAC theory, multiple models will be built based on the uncertainties of controlled model's parameters and structure. Multiple controllers will be designed according to multiple element models. According to the given switching mechanism, the model with most appropriate parameters to describe the plant will be decided, and the current controller will be switched to the controller corresponding to the best model $[22,23]$. Therefore, in order to better meet the requirements of actual production, we introduce the idea of multimodel adaptive control into iterative learning control and design multiple model iterative learning control (MMILC) $[24,25]$. Before every iteration, the best model is decided by the index switching function and corresponding controller based on ILC learning law is activated; thus the number of iterations will be reduced and the quality of the weld will be improved greatly.

This paper is organized as follows. In Section 2, we formulate the problem of GTAW process and welding model. In Section 3, we design iterative learning controller and multiple model iterative learning controller and analyze the convergence of the algorithm. Simulation and analysis are performed based on the welding model by using ILC and MMILC in Section 4. Finally, conclusions are given in Section 5 .

\section{Analysis of GTAW Process Control Problem}

2.1. GTAW Process. GTAW is a kind of welding method in which tungsten rod is used as one electrode, base metal 


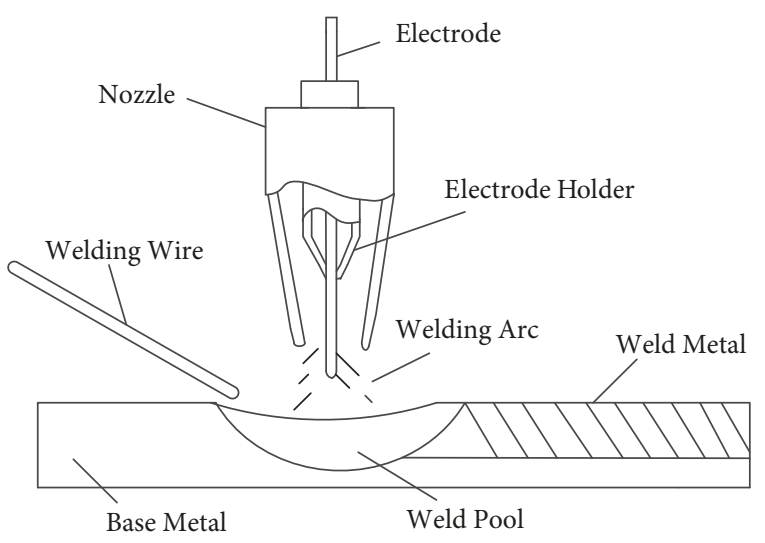

FIgURE 1: Schematic diagram of GTAW.

is used as the other electrode, and inert gas is used to protect the arc between the two electrodes. The schematic diagram of GTAW process is shown in Figure 1. The tungsten electrode is clamped on the electrode holder and protrudes from the welding gun nozzle. The welding workpiece is welded by an arc that generated between the end of the tungsten electrode and the welding workpiece. After the beginning of the welding process, the arc conducts heat to the welded workpiece, which causes the temperature of the weld workpiece to rise rapidly, and then the weld workpiece is partially melted to form a weld pool. During the welding process, the nozzle emits protective gas around the tungsten electrode to avoid pollution to the welding process from the atmosphere. When it is necessary to fill the weld pool with metal, the welding wire can be fed into the weld pool at a certain speed from the front of the arc.

2.2. System Model of GTAW. In the welding process, fusion penetration is the precondition to ensure the quality of welding. While back weld width, that is, the back width of weld pool, is the most direct expression of fusion penetration, so we choose the back weld width as the output of welding system. Many factors can influence the thermodynamic process to a great extent in welding, such as welding speed, welding current, wire feed rate, arc length, etc. These factors affect weld penetration, the size of the front and back pool, weld reinforcement, etc. Therefore, in theory, the above parameters can be quantified as input variables of the welding system. However, considering the controllability of parameters and the actual situation of the control system, the welding current and the wire feed rate are selected as the system inputs.

The input-output dynamic characteristic model of GTAW system [26] is as follows:

$$
\begin{aligned}
y(t)= & a_{1} y(t-1)+a_{2} y(t-2)+a_{3} y(t-3) \\
& +a_{4} y(t-4)+a_{5} y(t-5)+b_{11} u_{1}(t-1) \\
& +b_{12} u_{1}(t-2)+b_{14} u_{1}(t-4)+b_{21} u_{2}(t-1) \\
& +b_{23} u_{2}(t-3)+b_{25} u_{2}(t-5),
\end{aligned}
$$

where $u_{1}(t), u_{2}(t)$ are input variables, and they denote welding current and wire feed rate, $y(t)$ is output variable, and it denotes the back weld width. This is a multi-input singleoutput system.

\section{Establishment of Controller and Convergence Analysis}

3.1. Single Model Iterative Learning Controller. For the dynamic characteristic model of GTAW system, a control input sequence $\left\{u_{1}(t), u_{2}(t), t \in[0, T-1]\right\}$ is given. Assuming $u_{1,2}(t)=0(t<0), y(t)=0(t \leq 0)$, the response of system (1) is as follows:

$$
\begin{aligned}
y(1)= & b_{11} u_{1}(0)+b_{21} u_{2}(0)=\boldsymbol{a}(0) \boldsymbol{u}(0), \\
y(2)= & a_{1} y(1)+b_{11} u_{1}(1)+b_{12} u_{1}(0)+b_{21} u_{2}(1) \\
= & a_{1}\left[b_{11} u_{1}(0)+b_{21} u_{2}(0)\right]+b_{11} u_{1}(1)+b_{12} u_{1}(0) \\
& +b_{21} u_{2}(1) \\
= & b_{11} u_{1}(1)+b_{21} u_{2}(1)+\left(a_{1} b_{11}+b_{12}\right) u_{1}(0) \\
& +a_{1} b_{21} u_{2}(0)=\boldsymbol{a}(0) \boldsymbol{u}(1)+\boldsymbol{a}(1) \boldsymbol{u}(0) .
\end{aligned}
$$

By analogy, we can get the following results:

$$
\begin{gathered}
y(3)=\boldsymbol{a}(0) \boldsymbol{u}(2)+\boldsymbol{a}(1) \boldsymbol{u}(1)+\boldsymbol{a}(2) \boldsymbol{u}(0), \\
\vdots \\
y(T)=\boldsymbol{a}(0) \boldsymbol{u}(T-1)+\cdots+\boldsymbol{a}(T-1) \boldsymbol{u}(0),
\end{gathered}
$$

where $\boldsymbol{a}(t)$ is a matrix consisting of one row and two columns, $\boldsymbol{a}(0)=\left[\begin{array}{ll}b_{11} & b_{21}\end{array}\right], \boldsymbol{a}(1)=\left[a_{1} b_{11}+b_{12} a_{1} b_{21}\right], \boldsymbol{u}(t)=$ $\left[\begin{array}{ll}u_{1}(t) & u_{2}(t)\end{array}\right]^{\mathrm{T}}$.

We define $\boldsymbol{U}=[\boldsymbol{u}(0), \boldsymbol{u}(1), \ldots, \boldsymbol{u}(T-1)]^{\mathrm{T}}, \boldsymbol{y}=$ $[y(1), y(2), \cdots, y(T)]^{\mathrm{T}}$, then model (1) can be written in matrix form as follows:

$$
y=L(a) U
$$

where

$$
\boldsymbol{L}(\boldsymbol{a})=\left[\begin{array}{cccc}
\boldsymbol{a}(0) & & & \\
\boldsymbol{a}(1) & \boldsymbol{a}(0) & & \\
\vdots & \vdots & & \\
\boldsymbol{a}(T-1) & \boldsymbol{a}(T-2) & \cdots & \boldsymbol{a}(0)
\end{array}\right] .
$$

Assuming that the impulse response sequence a of the system cannot be accurately known, ILC can be used to implement the tracking tasks. $k$ denotes the number of iterations; we have

$$
\begin{aligned}
\boldsymbol{U}_{k} & =\left[\boldsymbol{u}_{k}(0), \boldsymbol{u}_{k}(1), \ldots, \boldsymbol{u}_{k}(T-1)\right]^{\mathrm{T}}, \\
\boldsymbol{y}_{k} & =\left[y_{k}(1), y_{k}(2), \ldots, y_{k}(T)\right]^{\mathrm{T}} .
\end{aligned}
$$


At the $k$ th iteration, model (4) can be written as

$$
y_{k}=L(a) U_{k}
$$

The following learning laws are used to obtain the control sequence:

$$
\boldsymbol{u}_{k+1}(t)=\boldsymbol{u}_{k}(t)+\sum_{s=0}^{T-1} \boldsymbol{c}(s) e_{k}(t-s)
$$

where $\boldsymbol{c}(t)$ is gain coefficient, $t \in[0, T-1]$.

From (8), we have

$$
\begin{aligned}
& {\left[\begin{array}{c}
\boldsymbol{u}_{k+1}(0) \\
\boldsymbol{u}_{k+1}(1) \\
\vdots \\
\boldsymbol{u}_{k+1}(T-1)
\end{array}\right]} \\
& =\left[\begin{array}{c}
\boldsymbol{u}_{k}(0) \\
\boldsymbol{u}_{k}(1) \\
\vdots \\
\boldsymbol{u}_{k}(T-1)
\end{array}\right] \\
& +\left[\begin{array}{cccc}
c(0) & & & \\
c(1) & c(0) & & \\
\vdots & \vdots & & \\
c(T-1) & c(T-2) & \cdots & c(0)
\end{array}\right]\left[\begin{array}{c}
e_{k}(0) \\
e_{k}(1) \\
\vdots \\
e_{k}(T-1)
\end{array}\right]
\end{aligned}
$$

where

$$
\boldsymbol{L}(\boldsymbol{c})=\left[\begin{array}{cccc}
\boldsymbol{c}(0) & & & \\
\boldsymbol{c}(1) & \boldsymbol{c}(0) & & \\
\vdots & \vdots & & \\
\boldsymbol{c}(T-1) & \boldsymbol{c}(T-2) & \cdots & \boldsymbol{c}(0)
\end{array}\right]
$$

Then the ILC law (8) can be written as follows:

$$
\boldsymbol{u}_{k+1}=\boldsymbol{u}_{k}+\boldsymbol{L}(\boldsymbol{c}) \boldsymbol{e}_{k},
$$

where $\boldsymbol{e}_{k}=\boldsymbol{y}_{d}-\boldsymbol{y}_{k}, \boldsymbol{y}_{d}$ is the desired output of the system, $\boldsymbol{y}_{k}$ is the actual output of the $k$ th iteration, and $\boldsymbol{L}(\boldsymbol{c})$ is the learning gain matrix which will be chosen.

3.2. Multiple Model Iterative Learning Controller. The dynamic process of welding is a complex object with multiple variable, multiple linear, time-varying parameters, and many uncertain factors. For the time-varying characteristics of welding system parameters, we consider the jump of system parameters which is a special case of time-varying parameters. Using the idea of multimodel, we establish multiple models to cover all jump parameters and design multimodel iterative learning controller to improve the control accuracy of welding system in the case of parameters jump.

(1) Establishment of Multiple Welding Models and Their Corresponding Controllers

$$
M=\left\{M_{i} \mid i=1,2, \cdots, n\right\},
$$

where $M_{i}$ denotes $n$ welding models, and it can be written as

$$
\begin{aligned}
y(t)= & a_{1}^{i} y(t-1)+a_{2}^{i} y(t-2)+a_{3}^{i} y(t-3) \\
& +a_{4}^{i} y(t-4)+a_{5}^{i} y(t-5)+b_{11}^{i} u_{1}(t-1) \\
& +b_{12}^{i} u_{1}(t-2)+b_{14}^{i} u_{1}(t-4)+b_{21}^{i} u_{2}(t-1) \\
& +b_{23}^{i} u_{2}(t-3)+b_{25}^{i} u_{2}(t-5) .
\end{aligned}
$$

The corresponding controllers are denoted as follows:

$$
C=\left\{C_{j} \mid j=1,2, \cdots, m\right\},
$$

where $C_{j}$ denotes controllers corresponding to the model $M_{i}$, and the controller of each model can make the corresponding model achieve good control performance. Besides, each controller can guarantee the stability of the system. $C_{j}$ can be written as

$$
\boldsymbol{u}_{k+1}=\boldsymbol{u}_{k}+\boldsymbol{L}(\boldsymbol{c}) \boldsymbol{e}_{k}
$$

where $\boldsymbol{e}_{k}=\boldsymbol{y}_{d}-\boldsymbol{y}_{k}$.

\section{(2) Establishment of Index Switching Function}

$$
L_{i, k}=\sum_{m=1}^{T} \beta^{T-m}\left|e_{k}^{i}(m)\right|, \quad 0<\beta \leq 1,
$$

where $e_{k}^{i}(t)=y_{k}(t)-y_{k}^{i}(t), y_{k}(t)$ denotes the ideal output of the system, $y_{k}^{i}(t)$ denotes the output of the $i$ th model, and $\beta$ denotes weighting factor.

Remarks. By establishing multiple models, the transient response of the system can be improved and the number of iterations can be reduced.

The switching steps of MMILC are as follows:

(1) Multiple models, corresponding controllers, and index switching functions are established.

(2) At the end of the $k$ th iteration, the best model to describe the current environment will be selected based on index switching functions; i.e., model $M_{l(k)}$ is the best model, $l(k)$ is defined by

$$
l(k)=\arg \min _{1 \leq i \leq n} \sum_{m=1}^{T} \beta^{T-m}\left|e_{k}^{i}(m)\right| .
$$

(3) In the next iteration, it will switch to controller $C_{l(k)}$ based on model $M_{l(k)}$. 


\subsection{Convergence Analysis of System with Fixed Parameters}

Lemma 1 (see [20]). For $A \in C^{n \times n}$ with its eigenvalues $\lambda_{i}, i \in\{1,2, \cdots, n\}$, if $\max _{i \in\{1,2, \cdots, n\}}\left|\lambda_{i}\right|<\delta<1$, the following inequality can be guaranteed:

$$
\left\|A^{k}\right\|_{2}<\sigma \gamma^{k}
$$

where $\sigma$ is a positive constant and $\delta<\gamma<1$.

Proof. For an arbitrary positive $\varepsilon$, there is a compatible norm $\|\cdot\|_{*}$ which satisfies

$$
\|A\|_{*}<\max \left|\lambda_{i}\right|+\varepsilon<\delta+\varepsilon .
$$

Due to $\delta<1$, we can let the $\varepsilon$ satisfy $\delta+\varepsilon<1$, and let $\gamma=\delta+\varepsilon$, then

$$
\left\|A^{k}\right\|_{*}<\underbrace{\|A\|_{*} \cdots\|A\|_{*}}_{k}<\gamma^{k}<1 .
$$

From the functional analysis, compatible norm satisfies

$$
\left\|A^{k}\right\|_{2}<\sigma\left\|A^{k}\right\|_{*}
$$

where $\sigma$ is a positive number which is determined by the relationship between 2 -norm and $*$-norm.

Hence

$$
\left\|A^{k}\right\|_{2}<\sigma \gamma^{k}
$$

This completes the proof.

Theorem 2. The error $\boldsymbol{e}_{k}$ converges to zero exponentially for the arbitrary initial error $\boldsymbol{e}_{0}$ as $k \longrightarrow \infty$ if the coefficient $\boldsymbol{c}(0)$ satisfies

$$
|1-\boldsymbol{a}(0) \boldsymbol{c}(0)|<1 .
$$

Proof. From model (7) and ILC law (11), we have

$$
\begin{aligned}
\boldsymbol{e}_{k+1} & =\boldsymbol{y}_{d}-\boldsymbol{L}[\boldsymbol{a}] \boldsymbol{u}_{k+1} \\
& =\boldsymbol{y}_{d}-\boldsymbol{L}[\boldsymbol{a}] \boldsymbol{u}_{k}-\boldsymbol{L}[\boldsymbol{a}] \boldsymbol{L}[\boldsymbol{c}] \boldsymbol{e}_{k} \\
& =\{\boldsymbol{I}-\boldsymbol{L}[\boldsymbol{a}] \boldsymbol{L}[\boldsymbol{c}]\} \boldsymbol{e}_{k},
\end{aligned}
$$

where $\boldsymbol{L}[\boldsymbol{a}]$ is a matrix with $T$ rows and $2 T$ columns and $\boldsymbol{L}[\boldsymbol{c}]$ is a matrix with $2 T$ rows and $T$ columns.

From the equation of $\boldsymbol{L}[\boldsymbol{a}]$ and $\boldsymbol{L}[\boldsymbol{c}]$, we can easily show that the matrix $\boldsymbol{L}(\boldsymbol{a}) \boldsymbol{L}[\boldsymbol{c}]$ is a lower triangular Toeplitz matrix with $T$ rows and $T$ columns. So $\boldsymbol{I}-\boldsymbol{L}[\boldsymbol{a}] \boldsymbol{L}[\boldsymbol{c}]$ is also a lower triangular Toeplitz matrix, and its first column is given as follows:

$$
\boldsymbol{f}(\boldsymbol{c} \mid \boldsymbol{a})=\boldsymbol{I}_{T}-\boldsymbol{L}[\boldsymbol{a}] \boldsymbol{c},
$$

where $\boldsymbol{c}=[\boldsymbol{c}(0), \boldsymbol{c}(1), \ldots, \boldsymbol{c}(T-1)]^{\mathrm{T}}$ and $\boldsymbol{I}_{T}=\left[\begin{array}{llll}1 & 0 & \cdots & 0\end{array}\right]^{\mathrm{T}}$ is a $T$ dimensional vector, so we can easily know that all eigenvalues of the lower triangular Toeplitz matrix $I$ $\boldsymbol{L}[\boldsymbol{a}] \boldsymbol{L}[\boldsymbol{c}]$ are given by $1-\boldsymbol{a}(0) \boldsymbol{c}(0)$.
Taking $\boldsymbol{A}=\boldsymbol{I}-\boldsymbol{L}[\boldsymbol{a}] \boldsymbol{L}[\boldsymbol{c}]$, (24) can be written as

$$
\boldsymbol{e}_{k}=A \boldsymbol{e}_{k-1}=A^{k} \boldsymbol{e}_{0} .
$$

Taking norms for both sides of (26) to get

$$
\left\|\mathrm{e}_{k}\right\|=\left\|A^{k}\right\| \cdot\left\|\boldsymbol{e}_{0}\right\| \text {. }
$$

Taking $\lambda=1-\boldsymbol{a}(0) \boldsymbol{c}(0)$, according to Lemma 1 , if the eigenvalues of $\boldsymbol{A}$ satisfy $\max _{i \in\{1,2, \cdots, n\}}\left|\lambda_{i}\right|<\delta<1,\left\|A^{k}\right\|_{2}<$ $\sigma \gamma^{k}$ can be guaranteed. So (27) can be written as

$$
\left\|\mathrm{e}_{k}\right\|<\sigma \gamma^{k}\left\|\boldsymbol{e}_{0}\right\|
$$

where $\sigma$ is a positive constant, $\delta<\gamma<1$. So when $k \longrightarrow \infty$, we can obtain $\left\|\mathrm{e}_{k}\right\| \longrightarrow 0$.

This completes the proof.

\subsection{Convergence Analysis of System with Jumping Parameters}

Theorem 3. If the system parameters jump during the iterative learning process, the error $\boldsymbol{e}_{k}$ converges to zero exponentially for the arbitrary initial error $\boldsymbol{e}_{0}$ as $k \longrightarrow \infty$ if the following conditions can be satisfied

(1) $\left|1-\boldsymbol{a}(0) \boldsymbol{c}_{j}(0)\right|<1, j=1,2, \ldots, m$.

(2) System parameters jump among finite values.

Proof. The proof can be performed similarly as in the proof of Theorem 2.

Assuming that the system model parameters jump among $m$ values in the iterative learning process, (26) can be written as follows:

$$
\boldsymbol{e}_{k}=\left(\boldsymbol{A}_{0}^{k_{m}} \cdot \boldsymbol{A}_{1}^{k_{m-1}} \cdot \cdots \cdot \boldsymbol{A}_{m-1}^{k_{1}} \cdot \boldsymbol{A}_{m}^{k_{0}}\right) \boldsymbol{e}_{0},
$$

where $k_{0}+k_{1}+\cdots+k_{m-1}+k_{m}=k, k$ is iteration number.

Take norms for both sides of (29) to get

$$
\left\|\mathrm{e}_{k}\right\|=\left(\left\|A_{0}^{k_{m}}\right\| \cdot\left\|A_{1}^{k_{m-1}}\right\| \cdot \cdots \cdot\left\|A_{m-1}^{k_{1}}\right\| \bullet\left\|A_{m}^{k_{0}}\right\|\right)\left\|\boldsymbol{e}_{0}\right\| .
$$

From Lemma 1, if the eigenvalues of $A_{0}, A_{1}, \ldots, A_{m}$ satisfy $\max _{i \in\{1,2, \cdots, n\}}\left|\lambda_{i}\right|<\delta<1$, we have

$$
\begin{aligned}
& \left\|\boldsymbol{A}_{0}^{k_{m}}\right\| \cdot\left\|\boldsymbol{A}_{1}^{k_{m-1}}\right\| \cdot \cdots \cdot\left\|\boldsymbol{A}_{m-1}^{k_{1}}\right\| \cdot\left\|\boldsymbol{A}_{m}^{k_{0}}\right\| \\
& \quad<\sigma_{0} \gamma^{k_{m}} \cdot \sigma_{1} \gamma^{k_{m-1}} \cdot \cdots \cdot \sigma_{m-1} \gamma^{k_{1}} \cdot \sigma_{m} \gamma^{k_{0}} \\
& \quad=\left(\sigma_{0} \sigma_{1} \cdots \sigma_{m-1} \sigma_{m}\right) \cdot\left(\gamma^{k_{m}} \gamma^{k_{m-1}} \cdots \gamma^{k_{1}} \gamma^{k_{0}}\right) .
\end{aligned}
$$

Substitute (31) into (30); we obtain

$$
\left\|\mathrm{e}_{k}\right\|<\left(\sigma_{0} \sigma_{1} \cdots \sigma_{m-1} \sigma_{m}\right) \bullet\left(\gamma^{k_{m}} \gamma^{k_{m-1}} \cdots \gamma^{k_{1}} \gamma^{k_{0}}\right)\left\|\boldsymbol{e}_{0}\right\| .
$$

According to Lemma 1 , we can easily know that $\sigma_{0} \sigma_{1} \cdots$ $\sigma_{m-1} \sigma_{m}$ is a positive constant. If $m$ is a finite value, then $\sigma_{0} \sigma_{1} \cdots \sigma_{m-1} \sigma_{m}$ is a bounded positive constant. So when $k \longrightarrow \infty$, we can obtain $\gamma^{k_{m}} \gamma^{k_{m-1}} \cdots \gamma^{k_{1}} \gamma^{k_{0}} \longrightarrow 0$, then $\left\|\mathrm{e}_{k}\right\| \longrightarrow 0$.

This completes the proof. 


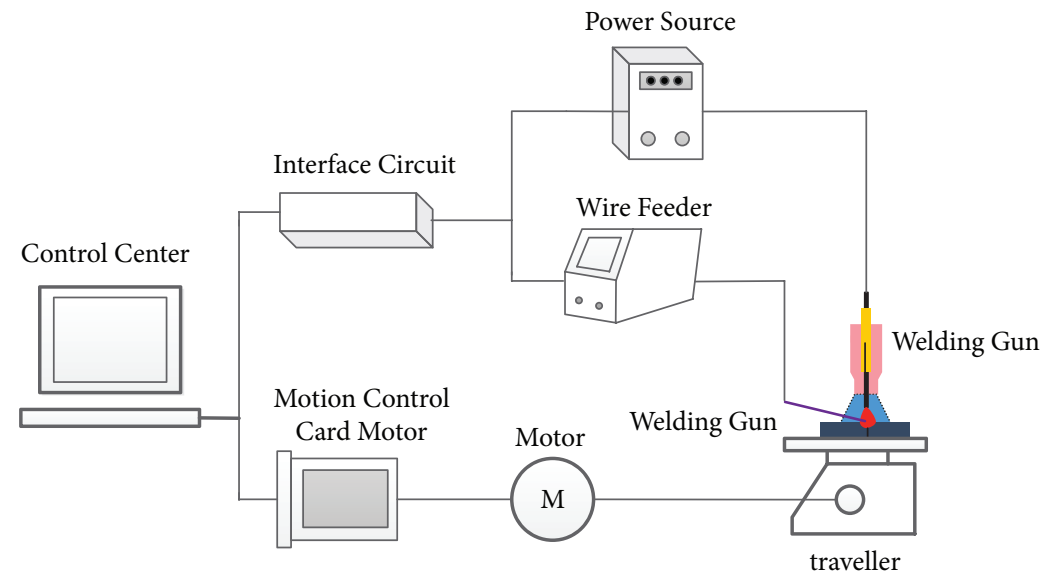

FIGURE 2: Structure diagram of GTAW system.

\section{Simulation Research and Analysis}

The mathematical model and model parameters of the welding system in this paper are derived from an actual welding system $[9,26,27]$. The structure of GTAW system is shown in Figure 2. The system mainly includes central control computer, welding power source, wire feeder, wire feeding control box, welding gun, gas flow controller, etc. Under the control of the central controller, the entire system can perform functions such as arc starting, welding, arc stopping, and walking. During the welding process, the back weld width is controlled by adjusting welding current and wire feed rate to ensure the welding quality. The welding current and the wire feed rate are controlled by adjusting the input voltage of the control end of welding power source. The peripheral equipment includes traveller and conveying mechanism. The conveying mechanism has two mutually perpendicular motion shafts, which can complete various curve movements in the plane.

The mathematical model of GTAW system is as follows:

$$
\begin{aligned}
y(t)= & a_{1} y(t-1)+a_{2} y(t-2)+a_{3} y(t-3) \\
& +a_{4} y(t-4)+a_{5} y(t-5)+b_{11} u_{1}(t-1) \\
& +b_{12} u_{1}(t-2)+b_{14} u_{1}(t-4)+b_{21} u_{2}(t-1) \\
& +b_{23} u_{2}(t-3)+b_{25} u_{2}(t-5),
\end{aligned}
$$

The system parameters are as follows:

$$
\begin{aligned}
& {\left[\begin{array}{lllll}
a_{1} & a_{2} & a_{3} & a_{4} & a_{5}
\end{array}\right]} \\
& \quad=[1.2245,-0.7935,0.45269,-0.23124,0.11518], \\
& {\left[\begin{array}{lll}
b_{11} & b_{12} & b_{14}
\end{array}\right]=[0.0085696,-0.3748,0.0039714],} \\
& {\left[\begin{array}{lll}
b_{21} & b_{23} & b_{25}
\end{array}\right]=[-0.16826,0.0023674,-0.079501] .}
\end{aligned}
$$

In order to show the effectiveness of our results, two cases are considered in this section.
4.1. ILC for Welding System with Constant Parameters. Taking $\left[\begin{array}{llll}\boldsymbol{c}(1) & \boldsymbol{c}(2) & \cdots & \boldsymbol{c}(T-1)\end{array}\right]=\left[\begin{array}{llll}\mathbf{0} & \mathbf{0} & \cdots & \mathbf{0}\end{array}\right]$, we have

$$
\boldsymbol{L}(\boldsymbol{C})=\left[\begin{array}{cccc}
\boldsymbol{c}(0) & & & \\
\mathbf{0} & \boldsymbol{c}(0) & & \\
\vdots & \vdots & \ddots & \\
\mathbf{0} & \mathbf{0} & \cdots & \boldsymbol{c}(0)
\end{array}\right]
$$

Substitute (35) into (11), the ILC law (11) can be written as

$$
\boldsymbol{u}_{k+1}(t)=\boldsymbol{u}_{k}(t)+\boldsymbol{c}(0) \boldsymbol{e}_{k}(t) .
$$

The matrix $\boldsymbol{a}(0)=\left[\begin{array}{ll}b_{11} & b_{21}\end{array}\right]=[0.0085696-0.16826]$ is given. Set the learning gain as $c(0)=\left[\begin{array}{ll}-0.05 & -0.2\end{array}\right]^{\mathrm{T}}$, then it follows that $|1-\boldsymbol{a}(0) \boldsymbol{c}(0)|=0.96<1$. The desired value of back weld width is given as $y_{d}(k)=6 \mathrm{~mm}$.

Figure 3 gives the tracking effect of back weld width at 5 th, 10th, and 30th iterations, respectively. It shows that the actual output of the system converges to the desired output quickly within 30 iterations. Moreover, the performance of the maximal tracking error is presented in Figure 4. It shows that the maximal tracking error converges to zero gradually with the increase of the number of iterations, which means that the back weld width will fully meet the desired value. It proves the effectiveness of ILC for GTAW process control.

4.2. Welding System with Jumping Parameters. The above simulation results in Section 4.1 are obtained under the condition that the welding system parameters are fixed. In this section, for the situation that the system parameters jump during the welding process, simulation and analysis are carried out. We assume that the real parameter $b_{21}$ of the welding model jumps during the welding process, and the jumping parameters are as follows:

$$
b_{21}= \begin{cases}-0.57 & k<5 \\ -0.37 & 5 \leq k \leq 10 \quad t \in[1, T] . \\ -0.16 & k>10\end{cases}
$$




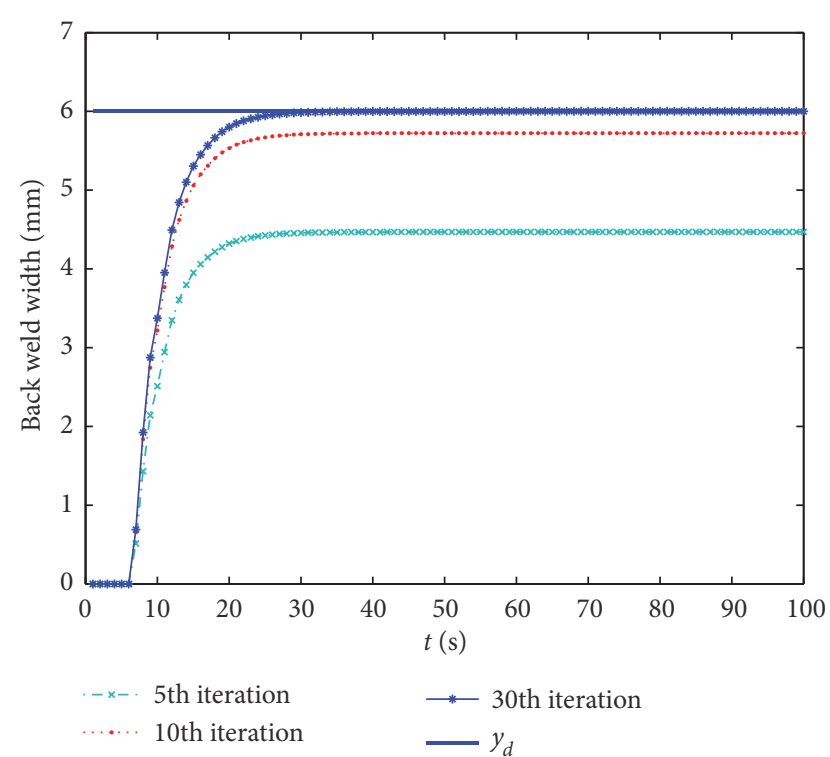

FIGURE 3: Tracking curve of back weld width.

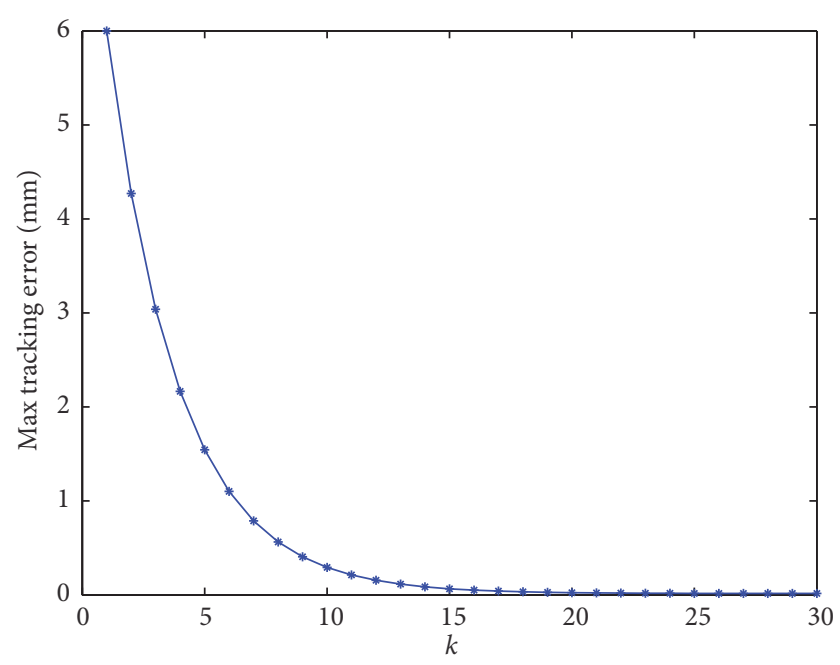

FIGURE 4: Maximal tracking error in iterative domain.

In modeling process, the model parameters we get are often inaccurate. We randomly select several estimation of $b_{21}$ around the true value for simulation. Three estimated values of the parameters are given as $b_{21}^{1}=-0.67, b_{21}^{2}=-0.42$, and $b_{21}^{3}=-0.17$, corresponding to model 1 , model 2 , and model 3. For system with jumping parameter, we consider two cases by using single model ILC and multiple model ILC.

Case 1 (single model ILC). For the system with jumping parameters, the simulation results using single model ILC are shown in Figures 5 and 6 . Figure 5 gives the tracking effect of back weld width at 8 th, 15th, 60th iterations, respectively. It shows that the tracking effect of the 15th iteration is worse than that of the 8th iteration because of the jump of system parameters, and after 60 iterations, the actual output converges to the desired output gradually. The performance

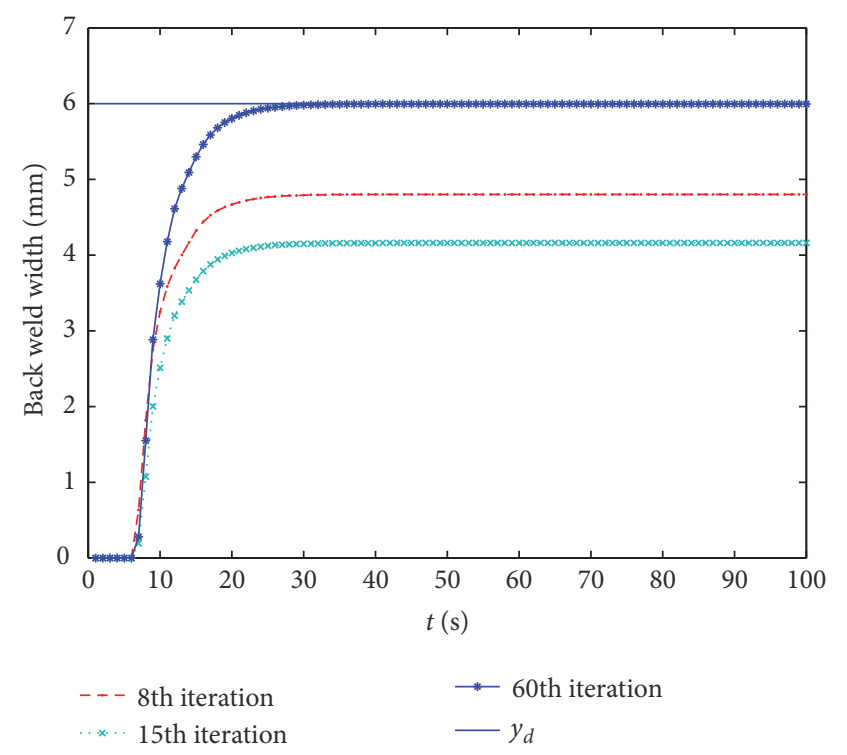

FIGURE 5: Tracking curve of back weld width.

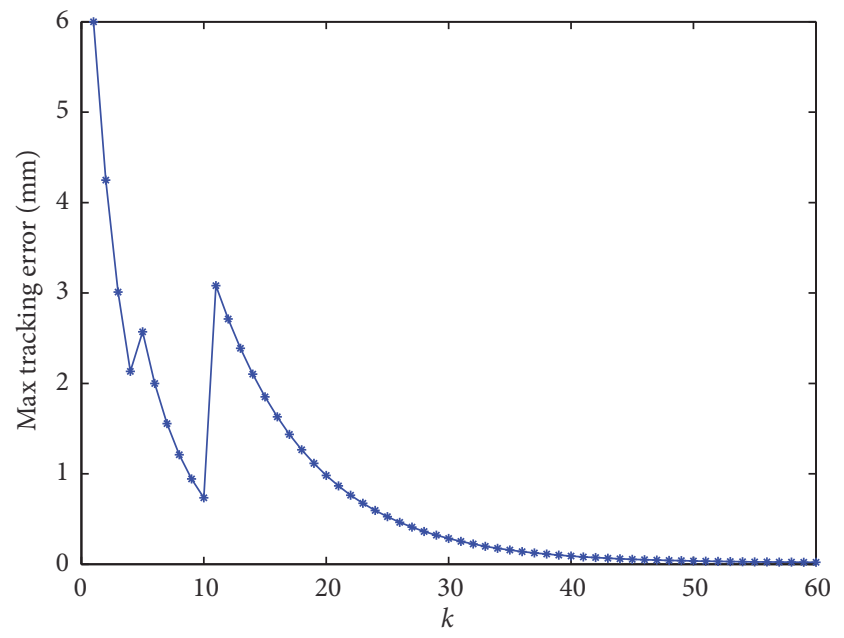

FIGURE 6: Maximal tracking error in iterative domain.

of the maximal tracking error in each welding process is presented in Figure 6. From Figure 6, we can see that the tracking error fluctuates greatly and the tracking effect is poor due to the jump of system parameters in the 5th and 10th iterations.

Case 2 (multiple model ILC). When multiple model ILC is applied to the welding system with jumping parameters, the simulation results which are shown in Figures 7, 8, and 9, and Figure 9 depict the switching procedure that how the best model is selected at every iteration when the parameter jumps, i.e., when the parameter $b_{21}$ changes from - 0.57 to 0.37 at the 5 th iteration, model 2 is calculated to be the precise one instead of model 1 , so model 2 is selected when the 6 th iteration begins. Through this control strategy, after about 25 iterations, the tracking error converges to zero gradually, and the performance of the maximal tracking error is shown in 


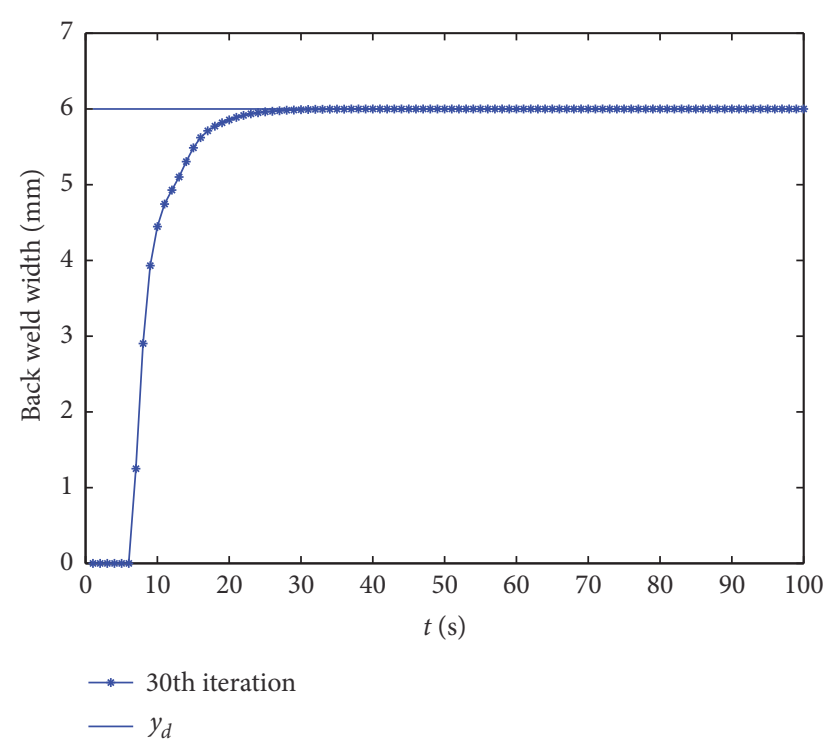

Figure 7: Tracking curve of back weld width.

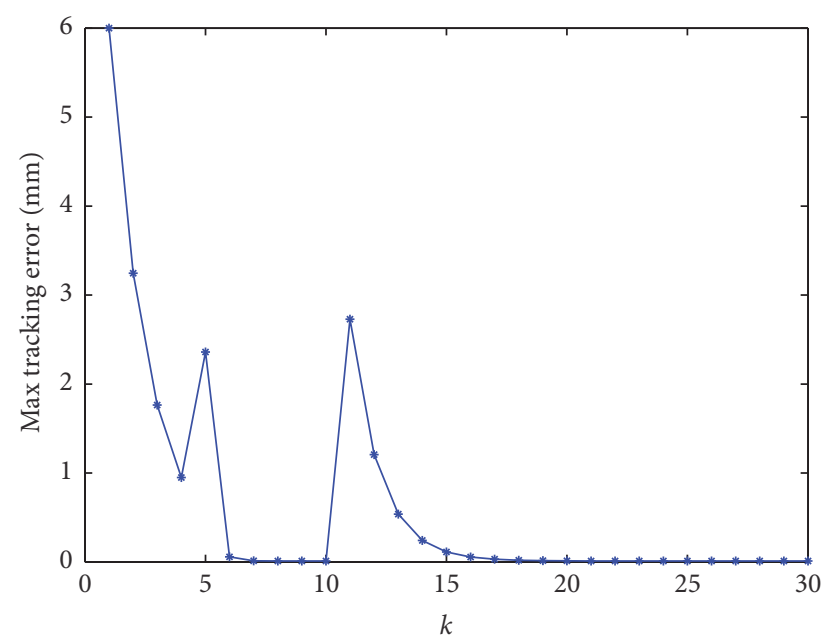

FIGURE 8: Maximal tracking error in iterative domain.

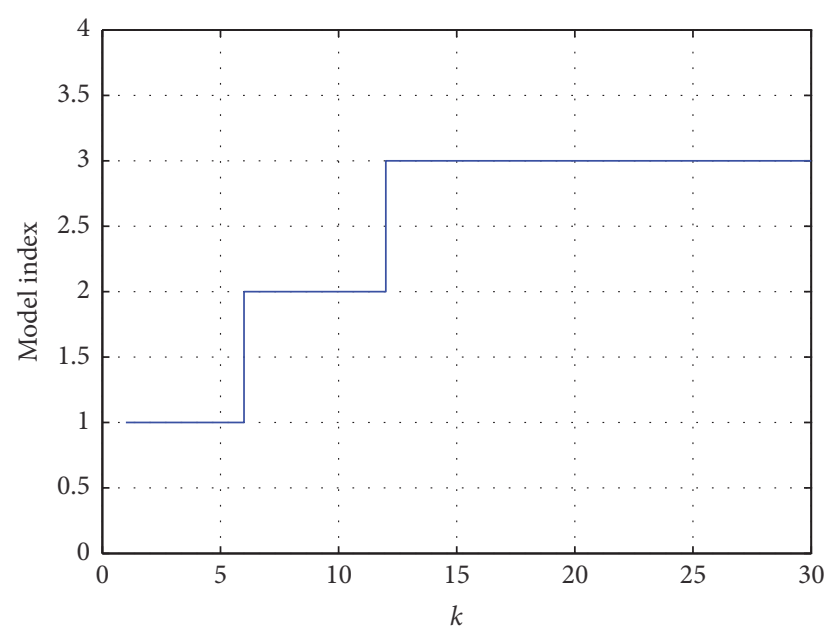

FIGURE 9: Switching procedure of model.
Figures 7 and 8. Compared with single model ILC, MMILC can decrease the number of iterations effectively and improve the tracking effect.

\section{Conclusions}

Aiming at the repeatability of automatic welding, the welding process is studied based on ILC algorithm in this paper. Considering that the welding process is a complex nonlinear process with multiple variable, multiple linear, and timevarying parameters and the system parameters jump easily due to external disturbances in the welding process, we combine MMAC with ILC. Firstly, the GTAW process and model are introduced. Then according to the model of GTAW process, an iterative learning controller and a multiple model iterative learning controller are designed. And the convergence of the algorithm is proved. The simulation results show the effectiveness of the algorithm.

\section{Data Availability}

The data used to support the findings of this study are included within the article.

\section{Conflicts of Interest}

The authors declare that they have no conflicts of interest.

\section{Acknowledgments}

This research is supported in part by the National Natural Science Foundation of China under Grants 61873006, 61473034, and 61673053, the Beijing Major Science and Technology Special Projects under Grant Z181100003118012, and National Key Research and Development Projects under Grants 2018YFC1602704 and 2018YFB1702704.

\section{References}

[1] K. Andersen, G. E. Cook, G. Karsai et al., "Artificial neural networks applied to arc welding process modeling and control," IEEE Transactions on Industry Applications, vol. 26, no. 5, pp. 824-830, 1990.

[2] K. S. Boo and H. S. Cho, "A self-organizing fuzzy control of weld pool size in GMA welding processes," Control Engineering Practice, vol. 2, no. 6, pp. 1007-1018, 1994.

[3] Y. M. Zhang, R. Kovacevic, and L. Li, "Adaptive control of full penetration gas tungsten arc welding," IEEE Transactions on Control Systems Technology, vol. 4, no. 4, pp. 394-403, 1996.

[4] Y. M. Zhang and R. Kovacevic, "Neurofuzzy model-based predictive control of weld fusion zone geometry," IEEE Transactions on Fuzzy Systems, vol. 6, no. 3, pp. 389-401, 1998.

[5] J. Wu, Y. Xue, Q. Wang, and S. Chen, "Identification of welding process and PID control in pulsed gas tungsten arc welding," Electric Machines and Control, vol. 6, no. 1, pp. 71-73, 2002.

[6] A. Hirai, Y. Kaneko, T. Hosoda, S. Yamane, and K. Oshima, "Sensing and control of weld pool by fuzzy-neural network in robotic welding system," in Proceedings of the 27th Annual 
Conference of the IEEE Industrial Electronics Society, pp. 238$242,2002$.

[7] Y. Lei, C. Zhang, X. Cheng, and X. Chen, "Application of Fuzzy Neural Network Controller Based on Genetic Algorithm in GTAW," Transactions of the China Welding Institution, vol. 24, no. 4, pp. 47-50, 2003.

[8] D. Quanying, C. Shanben, and L. Tao, "Inspection of weld shape based on the shape from shading," The International Journal of Advanced Manufacturing Technology, vol. 27, no. 7-8, pp. 667671, 2006.

[9] F. Lv, Study on Model-Free Adaptive Control of Weld Pool Dynamic Process in Pulsed GTAW Welding Pool, Shanghai Jiaotong University, Shanghai, China, 2008.

[10] C.-Y. Wu, P.-C. Tung, and C.-C. Fuh, "Development of an automatic arc welding system using an adaptive sliding mode control," Journal of Intelligent Manufacturing, vol. 21, no. 4, pp. 355-362, 2010.

[11] Y. K. Liu and Y. M. Zhang, "Model-Based predictive control of weld penetration in gas tungsten arc welding," IEEE Transactions on Control Systems Technology, vol. 22, no. 3, pp. 955-966, 2014.

[12] H. S. Ahn, Y. Chen, and K. L. Moore, "Iterative learning control: brief survey and categorization," IEEE Transactions on Systems Man and Cybernetics Part C, vol. 37, no. 6, pp. 1099-1121, 2007.

[13] D. Shen and Y. Wang, "Survey on stochastic iterative learning control," Journal of Process Control, vol. 24, no. 12, pp. 64-77, 2014.

[14] D.-H. Hwang, B. K. Kim, and Z. Bien, "Decentralized iterative learning control methods for large scale linear dynamic systems," International Journal of Systems Science, vol. 24, no. 12, pp. 2239-2254, 1993.

[15] J. van Zundert, J. Bolder, and T. Oomen, "Optimality and flexibility in iterative learning control for varying tasks," Automatica, vol. 67, pp. 295-302, 2016.

[16] O. Tutty, M. Blackwell, E. Rogers, and R. Sandberg, "Iterative learning control for improved aerodynamic load performance of wind turbines with smart rotors," IEEE Transactions on Control Systems Technology, vol. 22, no. 3, pp. 967-979, 2014.

[17] Y. M. Zhao, Y. Lin, F. Xi, and S. Guo, "Calibration-based iterative learning control for path tracking of industrial robots," IEEE Transactions on Industrial Electronics, vol. 62, no. 5, pp. 29212929, 2015.

[18] H. Ji, Z. Hou, and R. Zhang, "Adaptive iterative learning control for high-speed trains with unknown speed delays and input saturations," IEEE Transactions on Automation Science and Engineering, vol. 13, no. 1, pp. 260-273, 2016.

[19] K. S. Narendra and C. Xiang, "Adaptive control of discretetime systems using multiple modles," IEEE Transactions on Automatic Control, vol. 45, no. 9, pp. 1669-1686, 2000.

[20] K. S. Narendra and H. Zhou, "New concepts control using multiple models," IEEE Transactions on Automatic Control, vol. 57, no. 1, pp. 78-89, 2012.

[21] M. Huang, X. Wang, and Z. Wang, "Multiple adaptive control for a class of linear-bounded nonlinear systems," IEEE Transactions on Automatic Control, vol. 60, no. 1, pp. 271-276, 2015.

[22] X.-L. Li, C. Jia, D.-X. Liu, and D.-W. Ding, "Nonlinear adaptive control using multiple models and dynamic neural networks," Neurocomputing, vol. 136, pp. 190-200, 2014.

[23] X.-L. Li, C. Jia, K. Wang et al., "Trajectory tracking of non-linear system using multiple series-parallel dynamic neural networks," Neurocomputing, vol. 168, pp. 1-12, 2015.
[24] X. L. Li and W. Zhang, "Multiple model iterative learning control," Neurocomputing, vol. 73, no. 13-15, pp. 2439-2445, 2010.

[25] X. Li, K. Wang, and D. Liu, "An improved result of multiple model iterative learning control," IEEE/CAA Journal of Automatica Sinica, vol. 1, no. 3, pp. 315-322, 2014.

[26] Z. Hou and S. Jin, Model-Free Adaptive Control: Theory and Application, Science Press, Beijing, China, 2013.

[27] F. L. Lü, J. F. Wang, C. J. Fan, and S. B. Chen, "An improved model-free adaptive control with $\mathrm{G}$ function fuzzy reasoning regulation design and its applications," Proceedings of the Institution of Mechanical Engineers, Part I: Journal of Systems and Control Engineering, vol. 222, no. 8, pp. 817-828, 2008. 


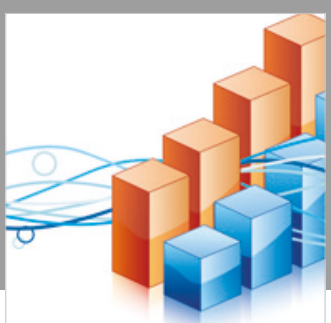

Advances in

Operations Research

\section{-n-m}
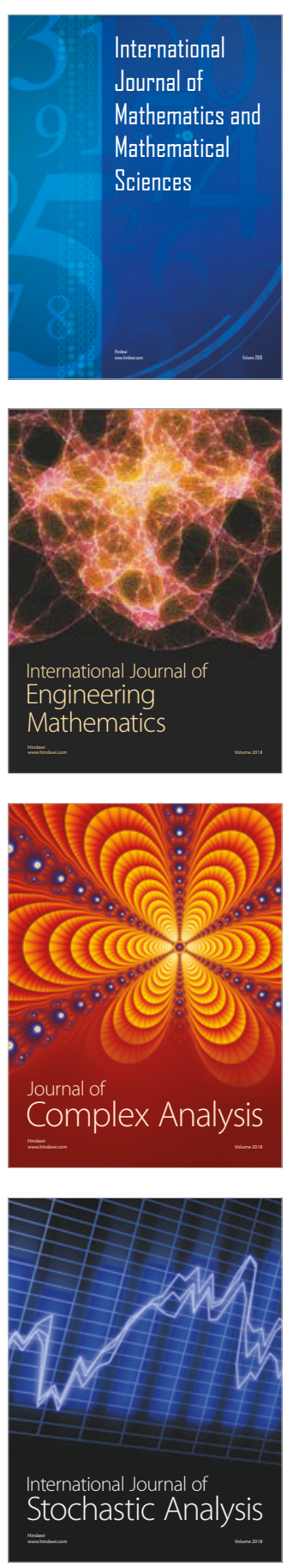
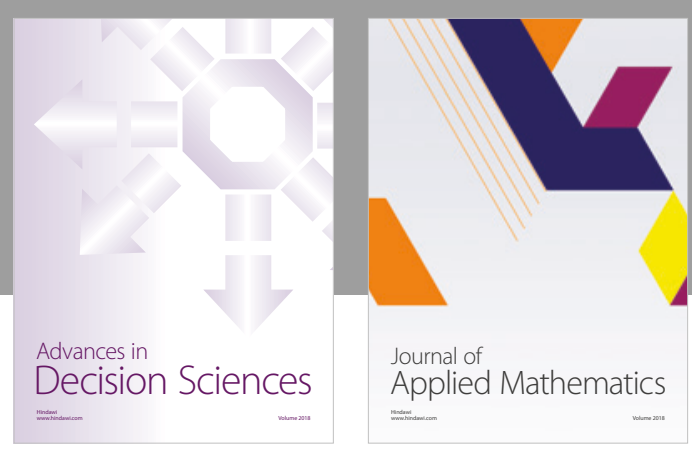

Journal of

Applied Mathematics
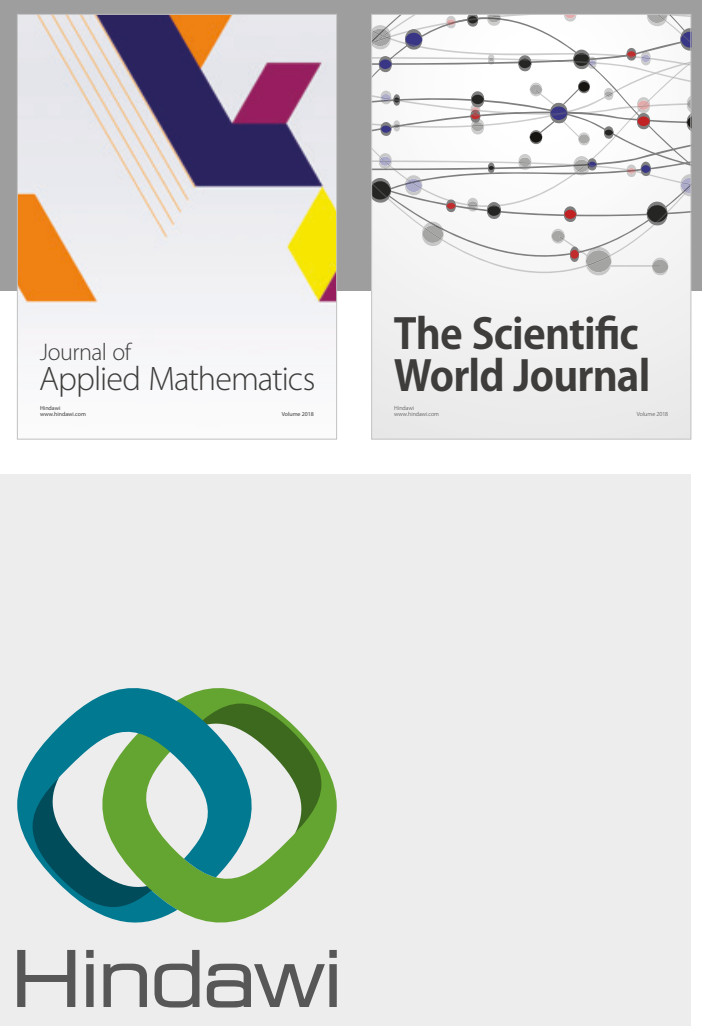

Submit your manuscripts at

www.hindawi.com

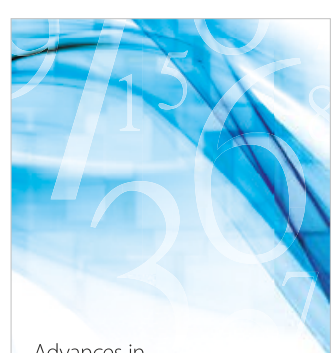

Advances in
Numerical Analysis
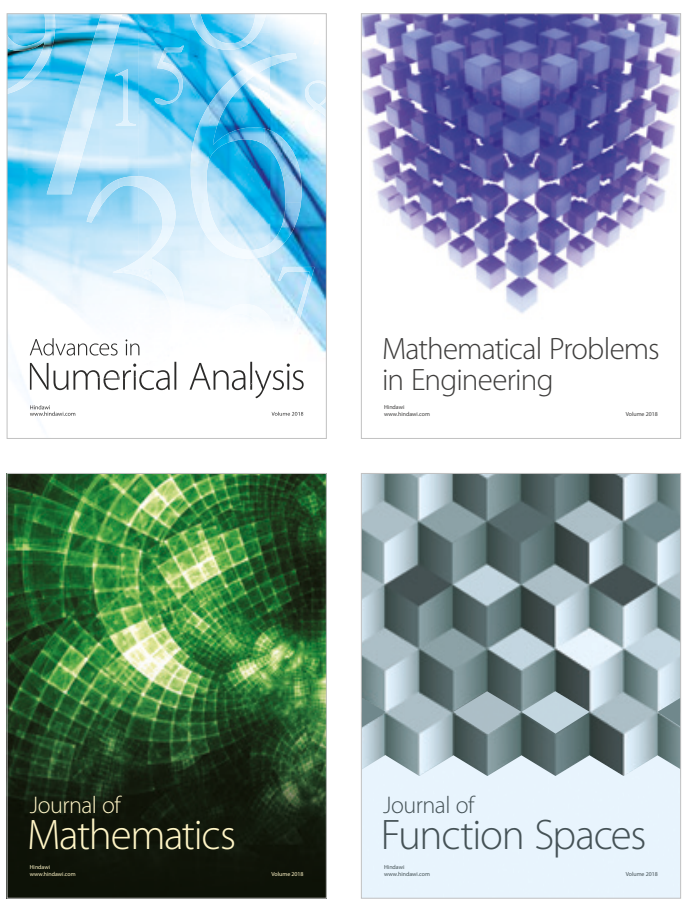

Mathematical Problems in Engineering

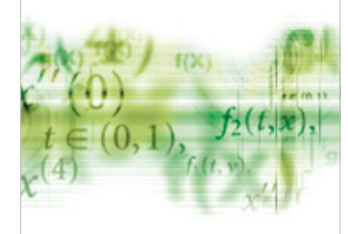

International Journal of

Differential Equations

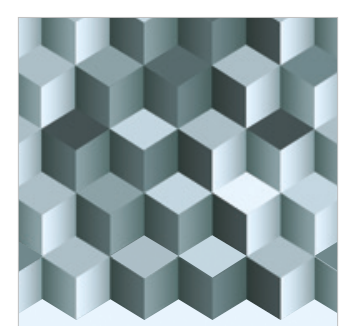

Journal of

Function Spaces

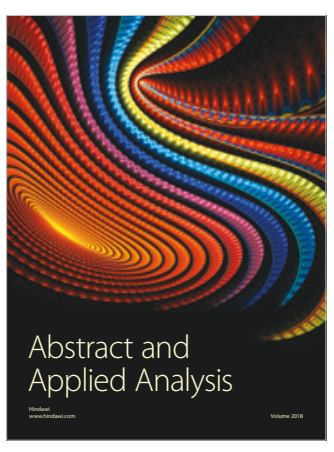

The Scientific

World Journal

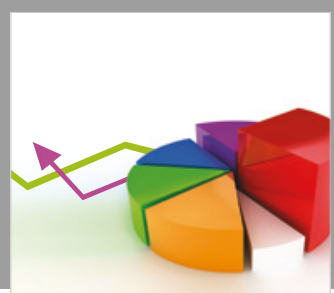

Journal of

Probability and Statistics
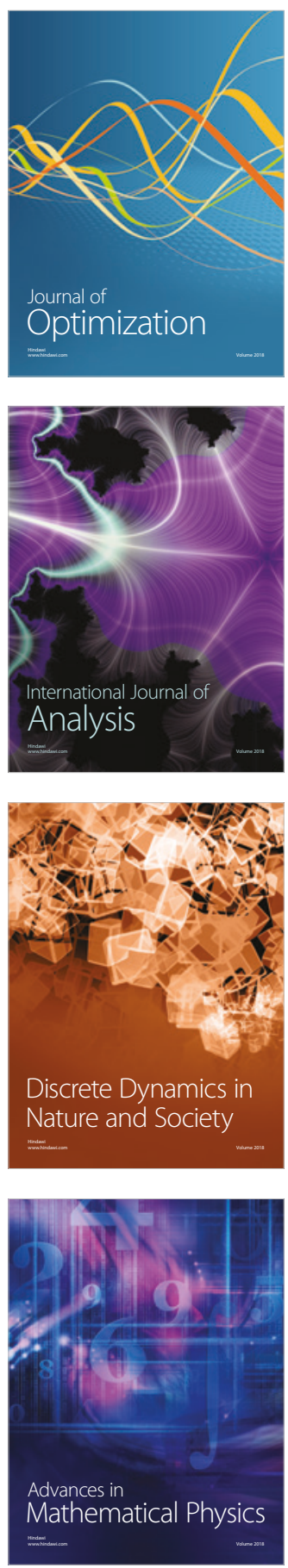